\title{
Heavy quarkonium in the saturated environment of high-multiplicity $p p$ collisions
}

\author{
B. Z. Kopeliovich $\odot,{ }^{1}$ H. J. Pirner, ${ }^{2}$ I. K. Potashnikova, ${ }^{1}$ K. Reygers, ${ }^{3}$ and Iván Schmidt ${ }^{1}$ \\ ${ }^{1}$ Departamento de Física, Universidad Técnica Federico Santa María, \\ Avenida España 1680, Valparaíso 2390123, Chile \\ ${ }^{2}$ Institute for Theoretical Physics, University of Heidelberg, Heidelberg 69120, Germany \\ ${ }^{3}$ Physikalisches Institut, University of Heidelberg, Heidelberg 69120, Germany
}

(Received 23 October 2019; accepted 21 February 2020; published 17 March 2020)

\begin{abstract}
High-multiplicity $p p$ collisions exhibit features, traditionally associated with nuclear effects. Coherence motivates to treat high-multiplicity $p p, p A$, and $A A$ collisions on an equal footing. We rely on the phenomenological parametrization for mean multiplicities of light hadrons and $J / \psi$, assuming their linear dependence on $N_{\text {coll }}$ in $p A$ collisions. The results of this approach underestimate the recently measured production rate of $J / \psi$ at very high hadronic multiplicities. The linear dependence of $J / \psi$ multiplicity on $N_{\text {coll }}$ is subject to predicted nonlinear corrections, related to mutual boosting of the saturation scales in colliding dense parton clouds. A parameter-free calculation of the nonlinear corrections allows us to explain data for $p_{T}$-integrated yield of $J / \psi$ at high hadronic multiplicities. Calculations are in a good accord with data binned in several $p_{T}$ intervals as well. As was predicted, $\Upsilon$ and $J / \psi$ are equally suppressed at forward rapidities in $p A$ collisions. Consequently, their fractional multiplicities at forward rapidities in $p p$ collisions are equal as well, and their magnitude agrees with data.
\end{abstract}

DOI: $10.1103 /$ PhysRevD.101.054023

\section{INTRODUCTION}

The popular models of multiparticle production in $p p$ and $p A$ collisions are based on the eikonal multi-Pomeron exchange, or Glauber models. On the contrary to the widespread belief, the Glauber model contains no clue to the multiplicity distribution. It is a model for the elastic scattering amplitude, which is related to the total cross section by the unitarity relation. One can also calculate the total inelastic cross section; however, no relation between the multiple scattering terms in the elastic amplitude and inelastic processes follows from the Glauber model. The unitarity relations between each term in the Glauber elastic amplitude, expanded over multiple interactions, and the corresponding inelastic processes, were proposed by Abramovsky, Gribov, and Kancheli [1], known as AGK cutting rules.

The unitarity cut of the elastic scattering amplitude can be done simultaneously through several Pomerons, while the other uncut Pomerons play a role in absorptive corrections. Those corrections, also known as shadowing, lead to a substantial reduction of the total inelastic cross section. However, they cancel each other and do not affect

Published by the American Physical Society under the terms of the Creative Commons Attribution 4.0 International license. Further distribution of this work must maintain attribution to the author(s) and the published article's title, journal citation, and DOI. Funded by SCOAP ${ }^{3}$. the inclusive particle production cross section. This peculiar feature of absorption corrections to the inclusive cross section is known as AGK cancellation [1].

Relating different unitarity cuts of the elastic amplitude with multiplicity of produced particles (any species), one finds that multiplicity is proportional to the number of cut Pomerons, usually called number of collisions, $N_{\text {coll }}$. Therefore, the normalized multiplicities of light hadrons and of $J / \psi$, defined, respectively, as

$$
R_{h} \equiv \frac{d N_{h} / d y}{\left\langle d N_{h} / d y\right\rangle}, \quad R_{J / \Psi} \equiv \frac{d N_{J / \Psi} / d y}{\left\langle d N_{J / \Psi} / d y\right\rangle},
$$

are expected to be equal, $R_{J / \Psi}=R_{h}$, and proportional to $N_{\text {coll }}$.

This expectation is in apparent contradiction with data [2-5], presented below in Fig. 1, demonstrating a significantly steeper rise of $R_{J / \Psi}$ with hadron multiplicity in comparison with $R_{h}$. Moreover, it has been known since long time [6] that the simple relation $R_{h}=N_{\text {coll }}$, offered by the eikonal model, is strongly broken, and the multiplicity dependence on $N_{\text {coll }}$ for light hadrons produced in $p A$ collisions is usually parametrized as

$$
R_{h}=1+\beta_{h}\left(N_{\text {coll }}-1\right),
$$

with $\beta_{h}<1$ fitted to data. In the Glauber model, $N_{\text {coll }}$ is given by 


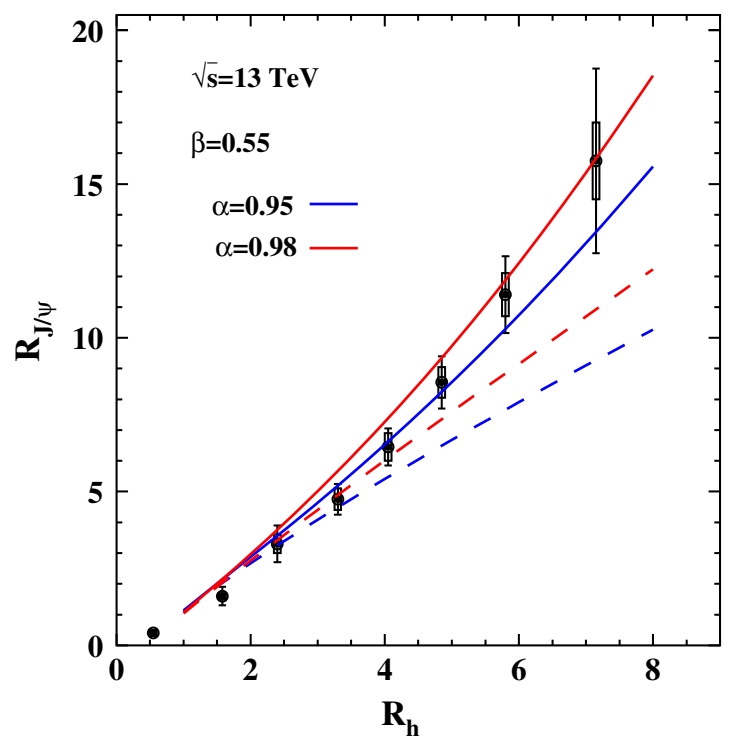

FIG. 1. Normalized multiplicity of $J / \Psi, R_{J / \Psi}$ vs normalized multiplicity of charged hadrons, $R_{h}$. Data are preliminary from ALICE [3-5] for $p p$ collisions at $\sqrt{s}=13 \mathrm{TeV}$ at for $y<0.9$. The dashed curves are calculated with Eqs. (5) and (7), while the solid curve incorporates the effect of mutually boosted saturation scale. In each pair, the upper and bottom curves are calculated with $\alpha=0.98$ and 0.95 , respectively.

$$
N_{\text {coll }}=A \frac{\sigma_{\text {in }}^{p p}}{\sigma_{\text {in }}^{p A}} .
$$

The analysis of available data on $p A$ collisions performed in [6] demonstrated consistency between different fits and no evidence for energy dependence of $\beta_{h}$. The multiplicity measured at $\sqrt{s}=5 \mathrm{TeV}$ in [7], $d N_{h}^{p A} / d y=$ $17.24 \pm 0.66$, which leads to $\beta_{h} \approx 0.55$. This is the value we rely upon in what follows.

The breakdown of the AGK cancellation is caused by coherence effects in gluon radiation [8]. The AGK rules [1] assume the Bethe-Heitler regime of particle production, which, e.g., leads to $n$-times higher rapidity density of multiplicity $d N_{h} / d y$ for $n$ cut Pomerons compared to a single cut. This regime is known to be broken by the Landau-Pomeranchuk effect of coherent radiation from multiple interactions. The reduction of gluon radiation caused by coherence is also known as gluon shadowing [9]. Only the accumulated mean transverse momentum of the radiated gluons keeps memory of multiple interactions, which lead to $p_{T}$ broadening of radiated gluons, usually called saturation or color-glass condensate [10].

Besides broken $N_{\text {coll }}$ dependence, Eq. (2), coherent production of quark-antiquark pairs also leads to a strong deviations from universality of $R_{h}$. At first glance, this might look surprising, because gluons are expected to hadronize at long distances, where no final state interaction is possible. Such a simplified interpretation of space time development is not correct.
As an example, $\bar{q} q$ production in DIS at small $x$ exhibits shadowing due to propagation and attenuation of the $\bar{q} q$ dipole through the whole target [11]. This shadowing is a higher twist, so it is weaker for heavy than for light quarks.

Production of $\bar{q} q$ by gluons is more involved; both the incoming gluon and produced $\bar{q} q$ interact with the target and screen each other. As a result, shadowing of quark production is also a higher twist and is described as propagation of an artificial three-body dipole $|\bar{q} q g\rangle$. Correspondingly, charm quarks [12] and charmonia [13-15] are shadowed considerably less than light quarks. This explains in particular the observed nonuniversality $R_{J / \Psi}>R_{h}$.

The effects of coherence possess a strong scale dependence for the relation between fractional multiplicity and number of collisions. If the popular parametrization Eq. (2) is applied to $J / \psi$,

$$
R_{J / \psi}=1+\beta_{J / \psi}\left(N_{\text {coll }}-1\right)
$$

one should expect a larger value of the parameter $\beta_{J / \psi^{*}}$.

Thus, from (2) to (4), we get

$$
\frac{R_{J / \Psi}-1}{R_{h}-1}=\frac{\beta_{J / \psi}}{\beta_{h}} .
$$

The difference between the parameters $\beta_{J / \psi}>\beta_{h}$ leads to a steeper rise of $R_{J / \Psi}$ in comparison with $R_{h}$, as is demonstrated by data.

The upper bound for the speed of rise for the ratio (5) is

$$
\frac{R_{J / \Psi}-1}{R_{h}-1} \leq \frac{1}{\beta_{h}} \approx 1.8 .
$$

The processes of least shadowing corrections, like production of Drell-Yan pairs, $\Upsilon, B$, and $D$ mesons, are expected to approach this bound.

We do not attempt here at theoretical evaluation of $\beta_{J / \psi}$ (though is doable [15]), but prefer to rely on a fit to data. Another popular parametrization applied to measure $A$ dependence of $J / \psi$ production is

$$
R_{J / \psi}^{A}=N_{\text {coll }} A^{\alpha-1},
$$

where the fitted parameter $\alpha$ turns out to be close to unity, implying a weak nuclear suppression of $J / \psi$. Expanding this expression in small parameter $1-\alpha \ll 1$, we can relate $\beta_{J / \psi}$ in (4) and $\alpha$,

$$
1-\beta_{J / \psi}=(1-\alpha) \ln A \frac{N_{\text {coll }}}{N_{\text {coll }}-1} \approx(1-\alpha) \ln A,
$$

assuming a large number of collisions.

The exponent $\alpha$ in (7) also does not expose a strong energy dependence. It was accurately measured at $\alpha=0.95$ in the fixed target experiment E866 at $\sqrt{s}=40 \mathrm{GeV}$ [16]. 
This value agrees with $\alpha=0.9-0.98$ measured at the midrapidity at $\sqrt{s}=5.02 \mathrm{TeV}[17,18]$, or interpolated between forward and backward rapidities [19-21].

Thus, according to (5), $R_{J / \Psi}$ rises linearly with increasing $R_{h}$. The dependence of $R_{J / \Psi}$ on $R_{h}$ shown in Fig. 1 by dashed curves was calculated at $\sqrt{s}=5.02 \mathrm{TeV}$, with $\beta_{h}=0.55$ and the interval values of $\alpha=0.95-0.98$, which we consider as the corridor of the current uncertainty in measured $A$ dependence of $J / \psi$ production. As we discussed above, neither $\beta_{J / \psi}$ nor $\beta_{h}$ demonstrates any significant energy dependencies; therefore, comparison of the results in Fig. 1 at different energies is legitimate.

While these results agree with data at $R_{h} \leq 4$, available at the time of publication of [22], new measurements at $\sqrt{s}=$ $13 \mathrm{TeV}$ and higher multiplicity up to $R_{h}=7$ [3-5] considerably exceed the predicted values of $R_{J / \Psi}$, as one can see in Fig. 1. Here we correct the previous calculations to the effect of boosting the saturation scales due to multiple interactions in two colliding dense partonic configurations. The effect proposed in [23] leads to a substantial enhancement of charmonium production [13]. Therefore, we expect this effect to pull the curves up in Fig. 1 improving agreement with data. Indeed, the magnitude of the boosting effect, evaluated below, well explains the steep rise of the $J / \psi$ rate with hadron multiplicity. At the same time, at forward rapidities, the boosting effect ceases, higher-twist shadowing suppresses the yield of $J / \psi$ in good accord with data.

The necessity of substantial increase of the saturation scale to explain the observed enhancement of $J / \psi$ production rate in rare high-multiplicity events was acknowledged in the analysis [24] done within the color glass condensate model. Such an enhancement of the saturation scale goes along with the boosting effect, employed here; however, the higher-twist effects were not considered in [24] while they dominate in our description.

Notice that the correlation of multiplicities of $J / \psi$ vs hadrons observed at forward rapidities is well described by the event generator Pythia [25]. However, this is not a parameter-free approach; the parameters of the generator are adjusted to data to be explained.

The models proposed to explain high-multiplicity events include percolation models [26,27], based on assumptions for poorly known nonperturbative dynamics of final state interactions of strings and formation of color ropes. It has no relation to the initial state phenomena like shadowing or parton saturation.

\section{EFFECTS OF GLUON SATURATION}

Multiple interactions naturally lead to broadening of the transverse momentum distribution, which has been calculated in $[28,29]$ for proton-nucleus collisions in agreement with data and predicted for high-multiplicity $p p$ collisions in [22]. In the leading order, broadening is proportional to the number of collisions with known coefficient,

$$
\Delta p_{T}^{2}=\frac{9 C(E)}{2 \sigma_{\text {in }}^{p p}}\left(N_{\text {coll }}-1\right),
$$

where $N_{\text {coll }}$ is related to $R_{h}$ by Eq. (4).

The energy-dependent factor $C(E)$ in (9) controls the cross section $\sigma_{\bar{q} q}(r)$ of interaction of a small size $r$ colorless dipole of energy $E$ (in the nuclear rest frame) [28],

$$
C(E)=\left.\frac{1}{2} \vec{\nabla}_{r_{1}} \cdot \vec{\nabla}_{r_{2}} \sigma_{\bar{q} q}\left(\vec{r}_{1}-\vec{r}_{2}, E\right)\right|_{\vec{r}_{1}=\vec{r}_{2}}
$$

Notice that for broadening of gluons, which are responsible for $J / \psi$ production, the pQCD factor $9 / 4$ is introduced in (9).

Since parton model interpretation of high-energy processes is not Lorentz invariant (only observable are), broadening of the $p_{T}$ distribution, which looks like a result of multiple interactions in the nuclear rest frame, is interpreted as saturation of small- $x$ parton density in the nucleus in its infinite momentum frame. Moreover, the value of the saturation scale is directly related to the magnitude of the broadening, $Q_{s}^{2}=\Delta p_{T}^{2}$ [29].

Apparently, the additional kicks gained by the parton from multiple collisions increase the effective scale of the process $Q^{2} \Rightarrow Q^{2}+\Delta p_{T}^{2}[13-15,23,30]$. As a result of Dokshitzer-Gribov-Lipatov-Altarelli-Parisi (DGLAP) evolution, the nuclear gluon density $g_{A}\left(x, Q^{2}+\Delta p_{T}^{2}\right)$ turns out to be suppressed at large $x$, but enhanced at small $x$ [23].

Thus, the rate of $J / \psi$ production in high-multiplicity $p p$ collisions turns out to be enhanced by gluon saturation and rises with $R_{h}$ steeper in comparison with Eq. (5).

In $p A$ collisions, only the projectile proton undergoes multiple interactions, which modify its unintegrated parton distribution function (UPDF), while the UPDFs of bound nucleons remain unchanged [23]. In the case of $A A$, or high-multiplicity $p p$ collisions, the interaction becomes symmetric, both assembles of colliding constituents are subject to multiple interactions, increasing their partonic content at small $x_{1,2}$. On the other hand, multiple interactions in a denser gluonic medium become more intensive, leading to a further increase of gluon density. Thus, a rising small- $x$ gluon density in one of the colliding protons, induced by multiple interactions at large $R_{h}$, stimulates stronger broadening of the partons in another colliding proton, which in turn leads to a further increase of gluon density in the first proton. Such a mutual boosting of the gluon densities and saturation scales in the colliding protons at large $R_{h}$ is similar to the rise of the saturation scales in colliding nuclei, which are described in more detail in [23], and satisfies the bootstrap equation, 


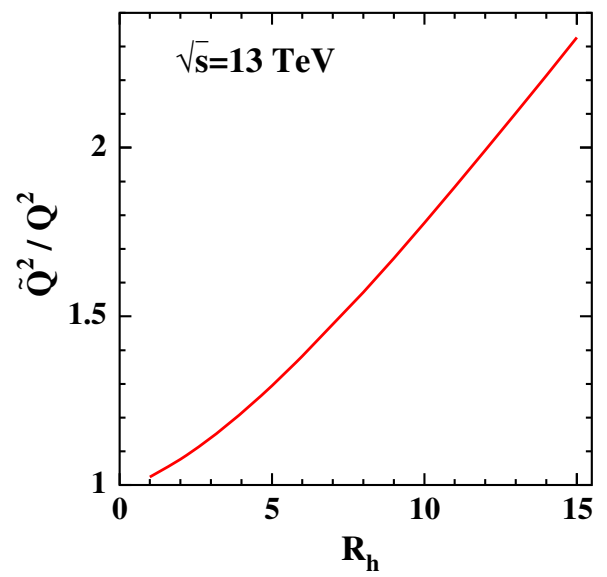

FIG. 2. Boosted saturation scale Eq. (11) in the proton relative to the original scale $Q^{2}=4 m_{c}^{2}$ vs fractional multiplicity of light hadrons.

$$
\tilde{Q}_{s}^{2}=\frac{3 \pi^{2}}{2} \alpha_{s}\left(\tilde{Q}_{s}^{2}+Q_{0}^{2}\right) x g\left(x, \tilde{Q}_{s}^{2}+Q_{0}^{2}\right) \frac{N_{c o l l}}{\sigma_{\mathrm{in}}^{p p}},
$$

where $N_{\text {coll }}$ is related to $R_{h}$ by (2). The characteristic scale $Q_{0}$ is the merging line between the nonperturbative and perturbative regimes of broadening. While the latter is described perturbatively, the former is not calculable, but relies on the dipole phenomenology [29],

$$
\frac{3 \pi^{2}}{2} \alpha_{s}\left(Q_{0}^{2}\right) x g\left(x, Q_{0}^{2}\right)=C(E),
$$

where $C(E)$ is given by Eq. (10) and $E=Q_{0}^{2} / 2 m_{N} x$. Such a strategy is similar to what is used in DGLAP-based analyses of data, except our evolution equation (11) is essentially nonlinear.

The saturated scale calculated with Eq. (11) for highmultiplicity $p p$ collisions relative to the characteristic scale $Q^{2}=4 m_{c}^{2}$ vs $R_{h}$ is shown in Fig. 2. At high multiplicities, the boosted scale significantly exceeds the original scale, generating via DGLAP evolution more gluons at small $x$ and a larger yield of $J / \psi$.

Notice that increase of the saturation scale hardly affects the hadron multiplicity $R_{h}$, because hadrons with low $p_{T}$, which give the main contribution to the multiplicity, are already produced with parton densities, which are at the unitarity bound. Therefore, $\beta_{h}$ shows no evidence for energy dependence.

\section{TRANSVERSE MOMENTUM DISTRIBUTION}

An increase of the scale of the process at small $x$ leads to a steep growth of the gluon density and as a result to enhanced production of $J / \psi$. Therefore, the relative production rate $R_{J / \psi}$ plotted in Fig. 1 by dashed curves should be corrected for the effects of saturation multiplying by the factor $g_{N}\left(x, \tilde{Q}_{s}^{2}+Q^{2}\right) / g_{N}\left(x, Q^{2}\right)$. The results are depicted in Fig. 1 by solid curves. Apparently, introduction of the

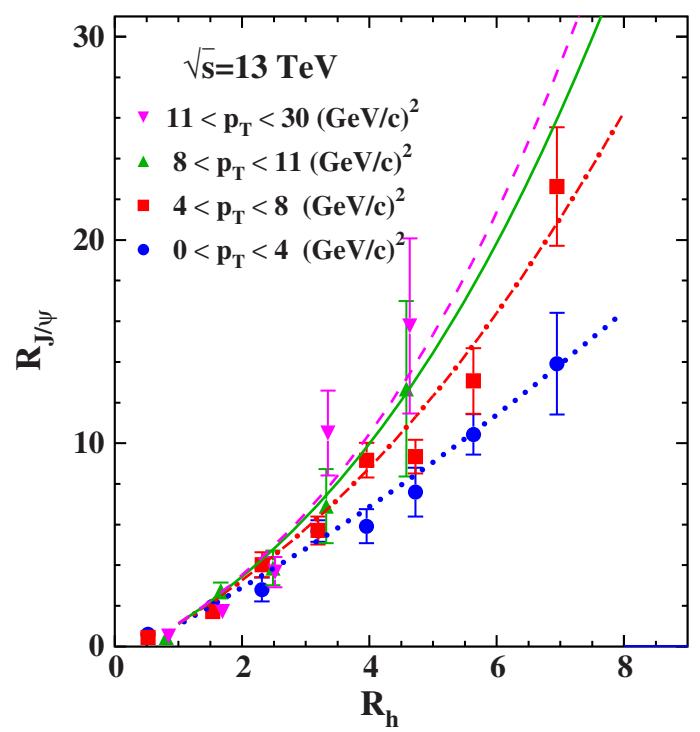

FIG. 3. Fractional multiplicity $R_{J / \Psi}$ vs fractional multiplicity of charged hadrons, $R_{h}$, for different intervals of $J / \psi$ transverse momentum $p_{T}$ indicated in the plot. Data are preliminary from ALICE [3-5]; the curves are calculated with Eq. (13) including the effects of saturation and mutual boosting of the saturation scale.

effects of saturation improved agreement with data at high multiplicities.

It is also instructive to compare our results and data in different intervals of transverse momentum $p_{T}$ os $J / \psi$,

$$
R_{J / \psi}\left(p_{T}\right)=\frac{\int_{p_{T}^{\min }}^{p_{\max }^{\max }} d p_{T} p_{T} d N_{J / \psi}^{p p}\left(R_{h}\right) / d y d^{2} p_{T}}{\int_{p_{T}^{\min }}^{p_{\max }} d p_{T} p_{T}\left\langle d N_{J / \psi}^{p p} / d y d^{2} p_{T}\right\rangle}
$$

Here the multiplicity of $J / \psi$ production in the nominator is taken at a given fractional multiplicity $R_{h}$ of light hadrons, while in the denominator the $J / \psi$ production rate is summed over hadron multiplicity. To perform integrations in (13), one needs to know the explicit form of $p_{T}$ dependence of the cross section. We rely on the popular parametrization,

$$
\frac{d N_{J / \psi}^{p p}}{d y d^{2} p_{T}}=\frac{d N_{J / \psi}^{p p}}{d y} \frac{1}{\left\langle p_{T}^{2}\right\rangle}\left(1+\frac{p_{T}^{2}}{(n-2)\left\langle p_{T}^{2}\right\rangle}\right)^{-n} .
$$

The mean transverse momentum squared can be extracted directly from data [20] applying a simple interpolation procedure. For $\sqrt{s}=13 \mathrm{TeV}$, we arrived at $\left\langle p_{T}^{2}\right\rangle=$ $11.72 \mathrm{GeV}^{2}$. Fixing this value and the shape (14), we fitted the remaining parameter at $n=3.2$.

We apply the same parametrization of the $p_{T}$ dependence to the numerator of (13), but increase the mean value of $p_{T}^{2}$ by broadening, $\left\langle p_{T}^{2}\right\rangle \Rightarrow\left\langle p_{T}^{2}\right\rangle+\Delta p_{T}^{2}$, which was calculated with Eq. (9). 


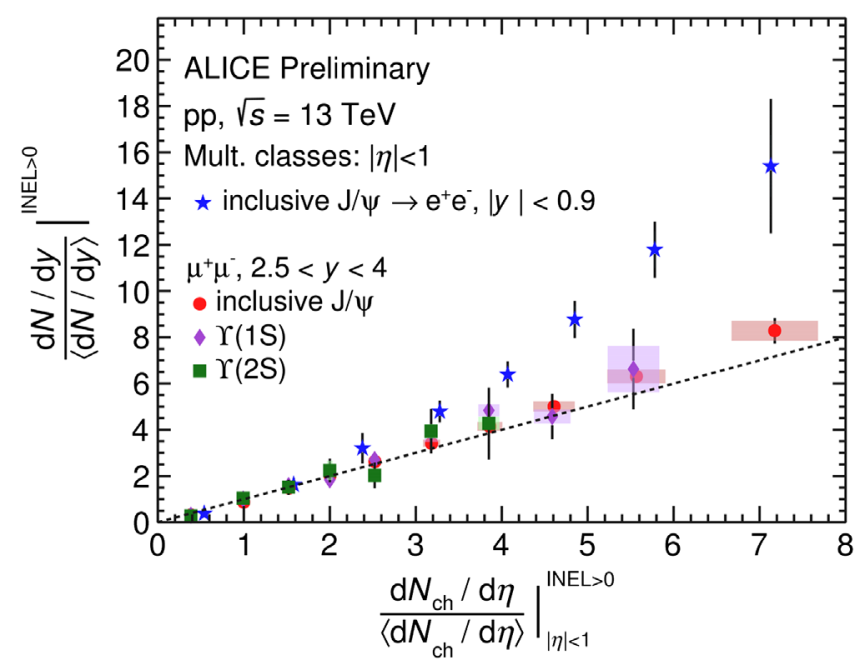

FIG. 4. Fractional multiplicities $R_{J / \Psi}$ and $R_{\Upsilon}$ at $2.5<y<4$ vs fractional multiplicity of charged hadrons, $R_{h}$ at $|\eta|<0$.9. Data are preliminary from ALICE [3-5] at $\sqrt{s}=13 \mathrm{TeV}$. The solid red curve is calculated with Eq. (5) with $\beta_{J / \psi}=\beta_{\Upsilon}=0.57$.

The data for $R_{J / \psi}$ measured in different $p_{T}$ intervals are depicted in Fig. 3 together with results of our calculations of Eq. (13) including the effects of saturation and mutual boosting of the saturation scale.

\section{IV. $J / \psi$ AND $\Upsilon$ AT FORWARD RAPIDITIES}

Data show that $J / \psi$ production rate in $p A$ collisions is more suppressed at forward than at the midrapidity $[16,19,21]$. For the interval $2.5<y<4$, measured in [19], the suppression factor $A_{\mathrm{eff}}^{J / \psi} / A \approx 0.65$, which corresponds to $\alpha=0.92$.

Although the radius of $\Upsilon$ is smaller than of $J / \psi$, and $\Upsilon$ is less suppressed at the midrapidity, the $\bar{b} b$ dipole cross section rises with energy faster than $\bar{c} c$ and $A_{\text {eff }}^{\mathrm{r}}$ steeply falls toward forward rapidities (see Fig. 4 in [13]). At rapidities $2.5<y<4$, higher-twist shadowing is predicted [13] to suppress $\Upsilon$ as much as $J / \psi, A_{\mathrm{eff}}^{\Upsilon} \approx A_{\mathrm{eff}}^{J / \psi}=0.65 A$.

Notice that the parameters $\beta_{J / \psi}=\beta_{\Upsilon}=0.57$ are only slightly above the hadronic value $\beta_{h}=0.55$, so the originally expected relation $R_{J / \psi}=R_{h}$ looks well satisfied. Of course, this does not mean restoration of the eikonal model; this is just a numerical coincidence.

Applying relation (5), we get $R_{J / \psi}=R_{\Upsilon}$ vs $R_{h}$ depicted by red solid curve in Fig. 4. Notice that it does not need to be corrected for the boosted saturation effects, because the correction is small at these rapidities (see Fig. 6 in [13]).

\section{SUMMARY}

High-multiplicity $p p$ collisions exhibit features which traditionally are associated with nuclear effects. Motivated by a long coherence time of interaction, we observe a close similarity between multiple interactions in $p p, p A$, and $A A$ collisions. In order to enhance multiple interactions in the former case, one should trigger on high multiplicity of produced hadrons, while nuclei allow to reach the same multiplicity easier, by increasing the number of collisions, Eq. (3). We employ the phenomenological description of the mean multiplicity in $p A$ collisions, Eq. (2), violating the AGK cutting rules. Fitted to data, the observed nuclear effects for $J / \Psi$ production enable one to predict the multiplicity dependence of the $J / \Psi$ production rate in $p p$ collisions. However, the linear relation Eq. (5) between the fractional multiplicities $R_{J / \psi}$ and $R_{h}$ underestimates the yield of $J / \psi$ at large hadron multiplicities [3-5].

Nevertheless, the linear dependence of the $J / \psi$ production rate on $N_{\text {coll }}$ is subject to nonlinear corrections, predicted in [23]. They are related to a mutual increase of the saturation scales in colliding nuclei [23], or two dense parton clouds. The parameter-free calculation of the nonlinear corrections allows us to explain the observed nonlinearity of $R_{J / \psi}$ vs $R_{h}$, as well as data binned in several $p_{T}$ intervals.

A sensitive testing of the present model can be made with recently measured [3-5] yield of $J / \psi$ at forward rapidities, which demonstrate similar fractional multiplicities $R_{J / \psi}=R_{h}$. We explain this result within our phenomenological description, while it might be a challenge for other available models. Besides, we predicted the same relation for heavy bottomium, $R_{J / \psi}=R_{\Upsilon}$, which is also in a good accord with data [3-5].

\section{ACKNOWLEDGMENTS}

We are thankful to Anton Andronic and Sarah Porteboeuf-Houssais for informative discussions and providing links to released new data. This work was supported in part by ANID-Chile FONDECYT Grants No. 1170319 and No. 1180232, by ANID PIA/APOYO AFB180002, and by USM internal project PI_LI_19_13. 
[1] V. A. Abramovsky, V. N. Gribov, and O. V. Kancheli, Yad. Fiz. 18, 595 (1973) [Sov. J. Nucl. Phys. 18, 308 (1974)].

[2] B. Abelev et al. (ALICE Collaboration), Phys. Lett. B 712, 165 (2012).

[3] D. Thakur For the ALICE Collaboration, Quarkonium production as a function of charged particle multiplicity in $\mathrm{pp}$ and $\mathrm{p}-\mathrm{Pb}$ collisions measured by ALICE at the LHC, Proc. Sci., HardProbes2018 (2018) 164; see also the talk at HardProbes2018, https://indico.cern.ch/event/ 634426.

[4] S. G. Weber (ALICE Collaboration), Measurement of $J / \psi$ production as a function of event multiplicity in pp collisions at $\sqrt{s}=13 \mathrm{TeV}$ with ALICE, Nucl. Phys. A967, 333 (2017).

[5] C. Jahnke (ALICE Collaboration), $\mathrm{J} / \psi$ production as a function of event multiplicity in $\mathrm{pp}$ collisions at $\sqrt{\mathrm{s}}=$ $13 \mathrm{TeV}$ using EMCal-triggered events with ALICE at the LHC, in Proceedings of the 14th International Workshop on Hadron Physics (Hadron Physics 2018) Florianopolis, Santa Catarina, Brazil (2018).

[6] W. Q. Chao, M. K. Hegab, and J. Hüfner, Multiparticle production in hadron-Nucleus collisions around 100-Gev, Nucl. Phys. A395, 482 (1983).

[7] B. Abelev et al. (ALICE Collaboration), Pseudorapidity Density of Charged Particles in $p+\mathrm{Pb}$ Collisions at $\sqrt{s_{N N}}=5.02 \mathrm{TeV}$, Phys. Rev. Lett. 110, 032301 (2013).

[8] B.Z. Kopeliovich, A. V. Tarasov, and A. Schafer, Bremsstrahlung of a quark propagating through a nucleus, Phys. Rev. C 59, 1609 (1999).

[9] B.Z. Kopeliovich, A. Schafer, and A. V. Tarasov, Nonperturbative effects in gluon radiation and photoproduction of quark pairs, Phys. Rev. D 62, 054022 (2000).

[10] L. D. McLerran and R. Venugopalan, Computing quark and gluon distribution functions for very large nuclei, Phys. Rev. D 49, 2233 (1994); 49, 3352 (1994); 50, 2225 (1994).

[11] B. Z. Kopeliovich, J. Raufeisen, and A. V. Tarasov, Challenges of nuclear shadowing in DIS, Phys. Lett. B 440, 151 (1998).

[12] B. Z. Kopeliovich and A. V. Tarasov, Gluon shadowing and heavy flavor production off nuclei, Nucl. Phys. A710, 180 (2002).

[13] B. Z. Kopeliovich, I. K. Potashnikova, H. J. Pirner, and I. Schmidt, Heavy quarkonium production: Nontrivial transition from pA to AA collisions, Phys. Rev. C 83, 014912 (2011).

[14] B. Z. Kopeliovich, I. K. Potashnikova, and I. Schmidt, Nuclear suppression of J/Psi: from RHIC to the LHC, Nucl. Phys. A864, 203 (2011).

[15] B. Z. Kopeliovich, I. Schmidt, and M. Siddikov, Suppression versus enhancement of heavy quarkonia in pA collisions, Phys. Rev. C 95, 065203 (2017).
[16] M. J. Leitch et al. (NuSea Collaboration), Measurement of $J / \psi$ and Psi-Prime Suppression in p-A Collisions at 800-GeV/c, Phys. Rev. Lett. 84, 3256 (2000).

[17] S. Acharya et al. (ALICE Collaboration), Prompt and nonprompt $J / \psi$ production and nuclear modification at midrapidity in $\mathrm{p}-\mathrm{Pb}$ collisions at $\sqrt{s_{N N}}=5.02 \mathrm{TeV}$, Eur. Phys. J. C 78, 466 (2018).

[18] S. Acharya et al. (ALICE Collaboration), Inclusive $\mathrm{J} / \psi$ production at mid-rapidity in pp collisions at $\sqrt{s}=5.02 \mathrm{TeV}$, J. High Energy Phys. 10 (2019) 084.

[19] B. B. Abelev et al. (ALICE Collaboration), $J / \psi$ production and nuclear effects in $\mathrm{p}-\mathrm{Pb}$ collisions at $\sqrt{S_{N N}}=5.02 \mathrm{TeV}$, J. High Energy Phys. 02 (2014) 073.

[20] J. Adam et al. (ALICE Collaboration), Rapidity and transverse-momentum dependence of the inclusive $\mathrm{J} / \psi$ nuclear modification factor in $\mathrm{p}-\mathrm{Pb}$ collisions at $\sqrt{s_{N N}}=5.02 \mathrm{TeV}$, J. High Energy Phys. 06 (2015) 055.

[21] S. Acharya et al. (ALICE Collaboration), Inclusive $\mathrm{J} / \psi$ production at forward and backward rapidity in $\mathrm{p}-\mathrm{Pb}$ collisions at $\sqrt{s_{\mathrm{NN}}}=8.16 \mathrm{TeV}$, J. High Energy Phys. 07 (2018) 160.

[22] B. Z. Kopeliovich, H. J. Pirner, I. K. Potashnikova, K. Reygers, and I. Schmidt, $J / \psi$ in high-multiplicity pp collisions: Lessons from pA collisions, Phys. Rev. D 88, 116002 (2013).

[23] B. Z. Kopeliovich, H. J. Pirner, I. K. Potashnikova, and I. Schmidt, Mutual boosting of the saturation scales in colliding nuclei, Phys. Lett. B 697, 333 (2011).

[24] Y. Q. Ma, P. Tribedy, R. Venugopalan, and K. Watanabe, Event engineering studies for heavy flavor production and hadronization in high multiplicity hadron-hadron and hadron-nucleus collisions, Phys. Rev. D 98, 074025 (2018).

[25] D. Thakur, S. De, R. Sahoo, and S. Dansana, Role of multiparton interactions on $J / \psi$ production in $p+p$ collisions at LHC energies, Phys. Rev. D 97, 094002 (2018).

[26] E. G. Ferreiro and C. Pajares, High multiplicity $p p$ events and $J / \psi$ production at LHC, Phys. Rev. C 86, 034903 (2012).

[27] E. G. Ferreiro and C. Pajares, Open charm production in high multiplicity proton-proton events at the LHC, arXiv: 1501.03381.

[28] M. B. Johnson, B. Z. Kopeliovich, and A. V. Tarasov, Broadening of transverse momentum of partons propagating through a medium, Phys. Rev. C 63, 035203 (2001).

[29] B. Z. Kopeliovich, I. K. Potashnikova, and I. Schmidt, Measuring the saturation scale in nuclei, Phys. Rev. C 81, 035204 (2010).

[30] B.Z. Kopeliovich, Puzzles of $J / \Psi$ production off nuclei, Nucl. Phys. A854, 187 (2011). 